\title{
El fragmentado mundo de la cultura y de la percepción estética: \\ Un acercamiento al discurso de Pierre Bordien
}

\author{
Salvador Orlando Alfaro \\ Departamento de Sociologia y Estudios Sociales \\ University of Regina, Canadá
}

La relación entre los sistemas de significados y la acción humana ha sido por mucho tiempo unos de los temas de discusión más importantes en la teoría de la cultura. Como puede encontrarse en los textos de discusión teórica, cada enfoque debe obligadamente tomar una posición con respecto a esta problemática. Algunos, como el interaccionismo simbólico y la etnometodología, asignan la prioridad al actor. Otras, como el neomarxismo de inspiración althusseriana, el funcionalismo de Parson y el estructuralismo, enfatizan el poder de los sistemas de significado en el control de los agentes. Se puede asegurar, que durante las últimas décadas del siglo xx la teoría de los estudios culturales centró su preocupación en la búsqueda de un punto medio entre estas dos posiciones opuestas.

El frogmentado mundo de la culturo y de la percepción estético 
Dentro de este panorama, una de las figuras intelectuales más influyentes en la teoría de la cultura y la investigación cultural ha sido, hasta su reciente fallecimiento, el sociólogo y crítico social francés Pierre Bourdieu. A través de todos sus trabajos culturales, Bourdieu persigue develar la mistificación causada por distorsiones ideológicas. Tres ideologías han sido objeto de su crítica permanente: la ideología del "ojo experto", la ideología del artista carismático y la ideología del sabor natural. En el terreno de la clásica crítica ideológica, muestra cómo estas tres actitudes sistemáticamente favorecen a la clase dominante. Para varios analistas de su obra, existe una equivalencia entre el enfoque de Bourdieu y el método que Marx utiliza en El Capital. En éste, Marx intenta demostrar que la ideología económica contiene vestigios religiosos o mágicos. Por su parte, el método de Bourdieu integra y utiliza la crítica de Marx en otra esfera de la producción en el período burgués: la de los bienes culturales (Fowler 1997).

Siguiendo a Bourdieu, uno puede afirmar que el enfoque prevaleciente en la producción cultural es básicamente la representación de una actitud esencialmente religiosa, de una operación misteriosa. Por lo tanto, la producción cultural es definida como la expresión de un genio transcendental, y es elevada tanto más allá de lo humano y más allá del análisis. Así, la recepción cultural es también naturalizada y de ese modo aparece como consecuencia de una distinción innata. Se deduce especialmente de la primera, la demanda que Bourdieu exige por un retorno a las relaciones sociales que definen los medios culturales, que por consiguiente no existe una "esencia" en el texto, es decir, ningun conjunto de reglas interpretativas que dicten los términos bajo los cuales un texto logre ambiguamente producir sus tesoros. En la perspectiva de Bourdieu, los cánones seculares de cultura consagrada todavían sirven como autoridad, incluso al precio de la violencia simbólica.

Bourdieu basa sus reflexiones en descripciones de novelas o poemas como mercancías abstractas o fetichizadas, en otras palabras, como productos que son vendidos en el mercado y percibidos como cosas, independientemente de las relaciones sociales específicas que las definen. Así como Marx mostró cómo la ideología de los economistas clásicos, con su Sagrada Trilogía de tierra, trabajo y capital, dependía de una manipulación mágica de categorías, así Bourdieu muestra que una magia similar toma lugar en las discusiones sobre los objetos consagrados en la cultura. 
De acuerdo con varios analistas de la teoría de la cultura, Bourdieu sus ofrece en su análisis uno de los mejores modelos sociológicos que pueden aplicarse al estudio de la cultura (Swartz 1997, Smith 2001). Ciertamente, su contribución a la teoría social incorpora una cantidad de temas claves en el debate teórico contemporáneo.

- Su planteamiento acerca del capital cultural y el habitus proporcionan un instrumento analítico poderoso en el estudio de la forma y la estructura de la cultura.

- Bourdieu puntualiza el papel relativamente autónomo de la cultura y las luchas culturales, al determinar el proceso, tanto de los resultados individuales como de los institucionales.

- Su trabajo es capaz de teorizar la estrecha relación que existe entre cultura y agencia.

En general, la teoría de Bourdieu es el resultado de un compromiso con las teorías objetivistas y subjetivistas que coexisten en el complejo mundo de la teoría social. Habiendo trabajado en ambos campos de la división metodológica y epistemológica, su mayor esfuerzo ha sido intentar articular una síntesis de la disputa entre ambas corrientes de pensamiento. Una manera de ilustrar su enfoque es comprender la manera en que aborda la relación entre consumo cultural y percepción estética en la época de la modernidad capitalista. Las siguientes páginas tienen el propósito de presentar esta relación, que, por lo demás, constituye una de las problemáticas más importantes en la prolífica obra del autor francés, además de incluir - a manera de conclusión - una breve discusión de algunos aspectos básicos de su sociología de la cultura.

\section{II}

Los estudios de Pierre Bourdieu se centran, básicamente, en los análisis de los consumos culturales, del arte y de la educación. Es por eso que, para comprender cómo se generan las categorías de percepción estética que determinan la experiencia subjetiva de lo bello desde Bourdieu, es necesario hacer referencia a los conceptos de: habitus, campo, capital cultural y codificación, entre otros.

El habitus es generado por las estructuras objetivas de éste y a su vez, genera las practicas individuales dando a la conducta esquemas de 
percepción, pensamiento y acción. El habitus sistematiza las prácticas y garantiza su coherencia con el desarrollo social. Por otra parte, tiende a reproducir las condiciones objetivas que le dieron origen, pero, ante contextos diferentes puede reorganizar las prácticas adquiridas y producir acciones transformadoras.

Los condicionamientos asociados a una clase particular de condiciones de existencia producen babitus, sistemas de disposiciones duraderas y transferibles, estructuras estructuradas predispuestas para funcionar como estructuras estructurantes, es decir, como principios generadores y organizadores de prácticas y representaciones que pueden estar objetivamente adaptadas a su fin sin suponer la buisqueda consciente de fines y dominio expreso de las operaciones necesarias para alcanzrlos, objetivamente 'reguladas' $y$ 'regulares' sin ser producto de la obediencia a reglas, $y$, a la vez que todo esto, colectivamente orquestadas sin ser el producto de la acción organizadora de un director de orquesta. (Bourdieu 1977: 72).

La construcción de la realidad social no opera en un vacío social, sino que está sometida a coacciones estructurales que a su vez son ya estructuras que tienen una génesis social. La construcción de la realidad no es un acto individual sino que está colectivamente organizada, sin ser por ello el producto de una acción organizada.

Es en este sentido que "la manifestación aparentemente, más libre de un sujeto, el gusto o, mejor, las categorías de percepción de lo bello, se dan como resultado del modo en que la vida de cada sujeto se adapta a las posibilidades estilísticas ofrecidas por su condición de clase. Así, la mirada del amante del arte del siglo xx es un producto de la historia, aunque surja bajo la apariencia de un don natural" (Bourdieu 1984). El gusto de una persona o grupo de personas se refiere al tipo de selección que este o estos realizan, tanto de objetos y propiedades como de las prácticas habituales que desempeñan. Es decir, el gusto se ve en la elección de determinados deportes, de determinadas comidas, libros, casas, lugares de recreación, etc.

"La obra de arte adquiere sentido sólo para aquel que posee un código específico para codificarla"(Bourdieu 1993:23). La adquisición de este código, de determinadas competencias estéticas es el producto de los efectos acumulados de la transmisión cultural asegurada por la familia y la escuela. Codificar es la manera de llevar a la práctica las 
reglas de un juego, de un determinado campo. Es la forma de ver, de clasificar, de percibir.

Los sistemas de percepción, en tanto sistemas de clasificación, de codificación, son objetivamente referidos — a través de los condicionamientos sociales que los han gestado- a una condición social. Todas las prácticas y todas las acciones están objetivamente armonizadas entre sí a través de un proceso inconsciente.

Esto nos lleva a hablar de otro término importante en la producción de Bourdieu, como es el campo. El campo serviría de mediación entre lo individual y lo social, es una red de relaciones objetivas entre posiciones, en un espacio donde los agentes pueden actuar pero a su vez se encuentran limitados por las mismas reglas que conforman el campo. El autor rechaza el concepto de "aparato ideológico" para no caer en la concepción de la escuela, los partidos políticos, la iglesia, etc., como "artefactos diabólicos" que obligan a los individuos a actuar de una forma determinada. Así, "un campo se vuelve un aparato cuando los dominantes tienen los medios para anular la resistencia y las reacciones de los dominados" (Bourdieu 1993:79).

Los campos se constituyen tanto por la existencia de un capital común como por la lucha por la apropiación de dicho capital. Para que se constituya un campo el capital —que puede ser económico, cultural, simbólico, social o politico- debe ser escaso y apreciado. Es importante aclarar que cuando se hace referencia al capital simbólico se está hablando de las formas que adoptan los distintos tipos de capital cuando son reconocidos como legítimos. El capital puede existir en estado objetivado (libros, propiedades) o bien -como en el caso del capital cultural - en estado incorporado al individuo (hábito), o institucionalizado (diploma académico).

Los gustos son el resultado de la confluencia entre el gusto objetivado del artista y el gusto del consumidor. Así, el sentido práctico del gusto es puramente negativo y realizado como rechazo de los otros gustos ya existentes.

Para comprender la producción de un artista es necesario ubicarlo - tanto al artista como a su producto- dentro de una red de relaciones que se establecen entre los agentes que están en contacto directo con la producción de la obra y la comunicación. Este sistema de relaciones no sólo comprende al artista, su obra y el público, sino que 
también se encuentran otros artistas, las otras obras, los críticos, los vendedores del arte, los dueños de las galerías de arte, etc., quienes determinan las condiciones específicas de producción y circulación de los productos culturales y constituyen el campo cultural.

La adquisición de competencias estéticas es el producto de los efectos producidos por la transmisión cultural tanto de la escuela como de la familia. Ambas contribuyen a formar una cultura legítima, por lo tanto la "mirada pura" es una invención histórica que es correlativa al surgimiento de un campo artístico capaz de imponer propias normas de producción y consumo.

Es necesario retomar el concepto de habitus, no como una serie azarosa de disposiciones, sino operado con la lógica de la práctica, de categorías simples y dicotómicas (adentro/afuera, bueno/malo, alto/ bajo, blanco/negro, común, vulgar, etc.) Estos principios de categorización que se desarrollan en el entorno inmediato de un(a) niño(a) pero que, en tanto principios reguladores a nivel inconsciente, pueden ser aplicados posteriormente a una gran variedad de campos. El babitus constituye el principio de percepción y apreciación de toda experiencia ulterior.

Pero, además, los objetos del mundo social se pueden percibir y decir de diferentes maneras porque, como los objetos del mundo natural, comportan siempre una parte de indeterminación y evanescencia que se debe a que aun las combinciones más constantes de propiedades, por ejemplo, sólo se basan en vínculos entre rasgos substituibles, así como que, en tanto objetos históricos, están sometidos a variaciones de orden temporal, ya que su propia significación, en la medida que está suspendida en el futuro, está en suspenso, en espera, y por lo tanto, relativamente indeterminada. (Bourdieu 1993: 76).

Los agentes o grupos de agentes se distribuyen en el campo social según el volumen de capital que poseen y las relaciones simbólicas que establecen en función de ese capital cultural. Las relaciones simbólicas deben interpretarse como los modos particulares de usar y consumir bienes.

Bourdieu señala en su celebrado trabajo Distinction: "Mi libro existe para llamar la atención sobre el hecho de que el acceso a la obra de arte requiere instrumentos que no están universalmente distribuidos. 
Y por lo tanto, los detentores de estos instrumentos se aseguran beneficios de distinción, beneficios que son más grandes en la medida en que sus instrumentos son más raros" (1984: 5).

En las luchas simbólicas por establecer el monopolio de la "nominación" o de la "mirada pura" legítima, los agentes compromenten todo su capital para imponer su vision del mundo. En este sentido, en el seno de la misma clase o entre las clases se dan diferencias en función de las artes (música, danzas, pintura, etc.) legítimas y de los usos que cada grupo hace de estas artes.

En este punto es necesario aclarar el concepto de clases sociales que maneja Bourdieu, el mismo no está dado solamente por el lugar que ocupan los agentes en las relaciones de producción.

"La clase social no se define por una propiedad (aunque se trate de las más determinantes, como el volumen de la estructura del capital ni por una suma de propiedades [propiedades de sexo, de edad, de origen social o étnico] —proporción de blancos y negros, por ejemplo; de indígenas y emigrados, etc.- de ingresos, de nivel de instrucción, etc.) ni mucho menos por una cadena de propiedades ordenadas a partir de una propiedad fundamental (la posición en las relaciones de producción en una relación de causa y efecto, de condicionante a condicionado, sino por la estructura de las relaciones entre todas las propiedades pertinentes, que confiere su propio valor a cada una de ellas y a los efectos que ejerce sobre las practicas" (Bourdieu 1984: 491).

Las relaciones simbólicas que se dan entre las clases muestran las diferencias que se reflejan en distinciones significantes. Las personas que componen una clase se determinan en relación con unos "índices concretos" de lo que les corresponde o no como clase. Estos índices son aplicados tanto en los objetos que consume como en la forma de apropiación de esos objetos y esto se da en una relación de poder.

Esto no quiere decir que un agente deba actuar en forma determinada por pertencer a una clase y no a otra, sino que el habitus funciona en forma inconsciente y por fuera del discurso y orienta a un sujeto a actuar y percibir según la posición que ocupa en el espacio social. El conocimiento que tiene este sujeto de su clase lo lleva a actuar de forma "razonable" en ese mundo que ya tiene divisiones, esquemas de clasificación que son, a su vez, distinciones. Las cosas que le resultan 
interesantes, que le "pertenecen" a un grupo de personas, no son más que interés por percibirlas como rasgos propios.

La diferencia entre las clases sociales, en el campo simbólico se establece - más que por la apropiación de bienes - en la forma de usarlos, ya que los mismos bienes pueden ser consumidos (y de hecho lo son) por personas de diferentes clases sociales.

En la introducción que García Canclini realiza del texto de Bourdieu, Sociología y Cultura, el autor distingue tres modos de producción que se encuentran en el mercado de bienes simbólicos:

Estos modos de producción cultural se diferencian por la composición de sus públicos (burguesia/clases medias/populares), por la naturaleza de las obras producidas (obras de arte/bienes y mensajes de consumo masivos) y por las ideologías político-estéticas que los expresan (aristocratismo esteticista/ascetismo y pretensión/pragmatismo funcional). (García Canclini, s.f.).

Se podría afirmar, entonces, que el mantenimiento de las clases sociales se da a partir de la distinción en las prácticas sociales y culturales ejercidas y una de las formas de distinción es el establecimiento, por parte de la clase dominante, del arte legítimo.

Como se dijo anteriormente, la distinción aumenta en la medida que los instrumentos necesarios para lograrla sean raros, escasos y que las pautas para codificarlos no estén distribuidas universalmente. Existe una estética particular para cada una de las clases sociales que distingue Bourdieu, así podemos hablar de una estética burguesa (según las estadísticas, son los que más concurren a los museos y los que le dedican más tiempo a la contemplación y admiración de las obras de arte), una estética de los sectores medios (este sector de la población cambia los museos por los shopping centers, los temas controvertidos por los personajes estereotipados, usan las técnicas de la fotografía para soleminizar los momentos más intensos de sus vidas), una estética popular (ésta se encuentra regida por la escasez de recursos económicos y por la necesidad de adquirir cosas prácticas y funcionales).

El análisis de Bourdieu da cuenta de las percepciones estéticas articulando los datos objetivos de las cosas sociales y las características que definen la inferioridad de los agentes. Para comprender esto con más facilidad, es conveniente remitirse al esquema que el autor realiza 
en el que encontrar una relación dialéctica entre objetividad dada por las condiciones de existencia objetivas de los agentes y las que se refieren a la subjetividad en las que se inscribe el babitus de un agente determinado como una estructura estructurada estructurante.

Esto acontece verdaderamente de esta manera cuando las categorias de percepción $y$ de acción que se ponen en funcionamiento en los actos individuales a través de los cuales se cumple la "voluntad" y el poder de la institución (ya se trate de las apreciaciones de un profesor a propósito de un ejercicio o de las preferencias de un alumno respecto de un establecimiento escolar o de una disciplina), tienen un acuerdo inmediato con las estructuras objetivas de organización porque son el producto de la incorporación de estas estructuras. (Bourdieu 1984:13,100).

Las categorías perceptivas no sólo se constituyen con las categorías materials que se pueden enumerar y medir sino que también cuentan con las propiedades simbólicas, las mismas propiedades materials vistas en relación, es decir como unas propiedades distintivas. La percepción del mundo social es el producto de una doble articulación: por un lado lo objetivo en el que la percepción está socialmente estructurada porque las propiedades atribuidas a los agentes se presentan en combinaciones que tienen posibilidades muy distintas, es decir, aquellos que tienen mayor manejo de la historia del arte más posibilidades existen de encontrarlos en exposiciones. Por otro lado, encontramos el costado subjetivo, los esquemas de percepción expresan el estado de las relaciones de poder simbólico. Estas dimensiones siempre compiten para producir un mundo común, para lograr un consenso.

\section{III}

El proyecto de Bourdieu ha tomado su forma en el transcurso de varios años. El sociólogo francés demuestra en sus análisis que los supuestos gustos naturales o individuales son, de hecho, construcciones sociales que han sido elaboradas por generaciones, a través del habitus. Bourdieu ha mostrado la re-emergencia de la distinción heredada en la relación diferencial en dos dimensiones fundamentales: en el conocimiento pedagógico (capital cultural) y en el área de la producción artística y el consumo.

El rogmentodo mundo de lo cultura y de lo percepción estético 
Bourdieu ha desarrollado una teoría de la práctica y un concepto del habitus que son adecuados a la complejidad de la realidad social. Su teoría de la práctica proporciona un avance genuino sobre las teorías sociales y culturales precedentes, la cual a pesar de tener una carga determinista, concibe a los agentes como entidades activas, como figuras que se transforman (Brubaker 1985). La síntesis que ofrece rechaza las alternativas subjetivistas y objetivistas, de igual modo que una corriente importante de la teoría de la cultura actual ha rehusado tomar la decisión de escoger entre dos paradigmas: estructuralismo y culturalismo. Al igual que el materialismo cultural de Raymond Williams, la teoría de Bourdieu se opone irreconciliablemente a la colonización total del sujeto por medio de la ideología, como en el caso del estructuralismo de Lévi-Strauss, y es la critica de este "idealismo nuevo" que establece una ruptura en su discurso, el cual busca introducir la práctica de los agentes en toda su dimensión. Al igual que Giddens, Bourdieu adoptó una actitud profundamente crítica de las promesas anunciadas por el estructuralismo (Giddens 1987: 195). Ambos sociólogos toman del materialismo histórico lo significativo del espacio y el tiempo en la vida social, y han criticado el estructuralismo por apoyarse exageradamente en la lingüística estructural como modelo para la transformación lingüística de la acción social. Sin embargo, Bourdieu proporciona un terreno más fertil para la sociología que la teoría de la estructuración de Giddens (Fowler 1997: 175).

Finalmente, unas palabras en torno a su orientación epistemológica. Exponiendo sus ideas en las primeras páginas de su Outline of Theory of Practice, Bourdieu ataca los planteamientos estructuralistas de lo social. Su principal argumento es que éstos nos llevan a un objetivismo distorsionado que nace gracias a la posición del científico social como observador. Desde la perspectiva de un agente externo era lógico construir mapas abstractos de los sistemas simbólicos y desarollar ideas de acerca de las reglas sociales. Desde esta posición, sólo hay un paso para afirmar que la vida social es dirigida por estas reglas y códigos en una forma determinista. Bourdieu argumenta que tales planteamientos niegan el papel de la agencia y la acción práctica en la vida social, especialmente con relación a factores de estrategia y emociones subjetivas como el honor y la verguenza. Al mismo tiempo, Bourdieu desea retener la comprensión en torno a los obstáculos cualitativos de la estructura social y la superioridad del conocimiento sociológico sobre 
el sentido común. En otras palabras, no cree que las explicaciones populares originadas en el sentido común puedan proporcionar una explicación adecuada de lo social (Bourdieu, Chamboderon y Passeron 1991).

En un esfuerzo de teorización sobre la relación entre estructura y agencia, sin reducir el análisis a un nivel u otro, Bourdieu argumenta que necesitamos una "sociología reflexiva". Esta sociología nos invita a pensar acerca de la manera en que nuestros modelos teóricos son influenciados por nuestras ubicaciones sociales y científicas y nos obliga a estar prevenidos de nuestros sesgos. Una vez hecho esto, empezaremos a movernos hacia adelante mediante la construcción de conceptos sociológicos que nos permitirán navegar en el sendero trazado por las construciones paradigmáticas del objetivismo y el subjetivismo. Para Bourdieu, el fruto de su propia sociología reflexiva fue un viraje sobre el objeto de análisis: el que va más allá tanto de la estructura como de la experiencia subjetiva hacia práctica social. Bourdieu comprende que las prácticas reflejan y reproducen tanto las relaciones sociales objetivas como las interpretaciones subjetivas de la realidad. Haciendo eco de su orientación epistemologica, Bourdieu —en uno de sus últimos textosexpresó su ambición de ser un intellectuel, y en tal sentido argumentó que los intelectuales tienen que jugar un papel importante en las arenas políticas y sociales en el contexto de las nuevas formas de dominación establecidas en la sociedad contemporánea (2001: 33-35).

\section{REFERENCIAS}

Bourdieu, P. (1977) Outline of Theory of Practice. Cambridge: Cambridge University Press.

(1984) Distinction: Londres: Routledge.

(1993) The Field of Cultural Production. Londres: Polity

Press.

(2001) Centre-feux 2. Pour un mouvement social europeén. Paris: Editions Raisons d'Agir.

Bourdieu, P., Chamboredon J.C. y Passeron, J. C. (1991) The Craft of Sociology. Epistemological Preliminaries. Berlin: Walter de Gruyter. 
Brubaker, R. (1985) "Rethinking Classical Sociological Theory: The Sociological Vision of Pierre Bourdieu", Theory and Society, 14: 74575.

Fowler, B. (1997) Pierre Bourdieu and Cultural Theory. Londres: Sage Publications.

García Canclini, N. "Introducción". P. Bourdieu Sociología y Cultura. Mimeo. $s / f$.

Giddens, A. (1987) "Structuralism", pp. 195-223, A. Giddens y B.Turner (eds.) Social Theory Today. Londres: Polity Press.

Smith, P. (2001) Cultural Theory. An Introduction. Malden, Mass.: Blackwell.

Swartz, D. (1997) Culture and Power: The Sociology of Pierre Bourdieu. Chicago: University of Chicago Press. 